\title{
Enfoques estratégicos del brand equity en las empresas que internacionalizan servicios de operación logística en Bogotá
}

Strategic Focuses of Brand Equity in Companies that Internationalize Logistics Operation Services in Bogota

\section{Enfoques estratégicos do brand equity nas empresas que internacionalizam serviços de operação logística em Bogotá}

\author{
Campo Elías López Rodríguez \\ Aníbal Acosta Molina ${ }^{\star *}$ \\ Alexandra Páez León ${ }^{* * \star}$ \\ Luisa María Sarmiento Páez \\ Andrea Tafur Serrano ${ }^{* \star * \star *}$
}

Fecha de recibido: 17 de abril de 2017

Fecha de aprobado: 13 de octubre de 2017

* Magíster en Gerencia Estratégica de Mercadeo de la Institución Universitaria Politecnico Grancolombiano. Docente del programa de Comercio Internacional de la Universidad ECCI, Bogotá, Colombia. Correoelectrónico: clopezr@ecci.edu.co

** Profesional en Comercio Internacional de la Universidad ECCI. Subdirector de Logistica en Texmoda S.A.S. Bogotá, Colombia. Correo electrónico: anibal.acosta@texmoda.com.co

*** Profesional en Comercio Internacional de la Universidad ECCI. Agente Logístico en Century Sports S.A.S. Bogotá, Colombia. Correo electrónico: alexandra.paez@sportline.com.co

***** Profesional en Comercio Internacional de la Universidad ECCI. Analista de Importaciones en Century Sports S.A.S. Bogotá, Colombia. Correo electrónico: luisa.sarmiento@sportline.com.co

****** Profesional en Comercio Internacional de la Universidad ECCI. Inside Sales en Ravago de Colombia S.A.S. Bogotá, Colombia. Correo electrónico: atafur@geochem.net 
Doi: http://dx.doi.org/10.12804/revistas.urosario.edu.co/empresa/a.5570

Para citar: López Rodríguez, C. E., Acosta Molina, A., Páez León, A., Sarmiento Páez, L.M., \&, Tafur Serrano, A. (2018). Enfoques estratégicos del brand equity en las empresas que internacionalizan servicios de operación logística en la ciudad de Bogotá. Universidad \& Empresa, 20135), 117-142. Doi: http://dx.doi.org/10. 12804/ revistas.urosario.edu.co/empresa/a.5570

\section{RESUMEN}

Este artículo de investigación científica explora la relación directa entre los enfoques estratégicos del brand equity y la importancia que tiene en las empresas que ofrecen servicios de operación logística, ya que es una herramienta que puede ayudar a las empresas, no solo a generar una identidad en el mercado logístico, sino a marcar una diferencia positiva entre su competencia, mostrando al mercado sus servicios de forma eficiente y con calidad; todo se realiza con el fin de estar en la mente de los clientes, para llegar a ser la opción que escojan siempre. Para este estudio se desarrolló una metodología cuantitativa, que persiguió describir el comportamiento de estos enfoques en los operadores logísticos de la ciudad de Bogotá, identificando hallazgos relevantes para este sector de la capital colombiana.

Palabras clave: Branding, valor de marca, operadores logísticos, internacionalización, servicios de operación logística.

\section{ABSTRACT}

This scientific research article explores the direct relationship between brand equity's strategic standpoints and the relevance that this aspect has for the companies offering logistic operation services. This concept is a tool that helps companies not only create an identity in the logistic market, but also make a great difference compared to their competition, showing efficient and quality services in the market. All of this is done in order to settle the brand into the customers' mind, and to turn into the chosen option by them. For this article, a quantitative methodology was carried out, that pursued to describe the behavior of those standpoints in the logistics operators in Bogota, identifying relevant findings for this sector in the Colombian capital city.

Keywords: Branding, branding value, logistics operator, internationalization, logistics operation services.

\section{RESUMO}

Este artigo de investigação científica explora a relação direta entre os enfoques estratégicos do brand equity e a importância que tem nas empresas que oferecem serviços de operação logística, devido a que esta é uma ferramenta que pode ajudar às empresas, não só a gerar uma identidade no mercado logístico, mas também a que possam marcar a diferença positiva entre a sua concorrência, mostrando ao mercado os seus serviços de forma eficiente e com qualidade; todo se realiza com o fim de estar na mente dos clientes, para chegar a ser a opção que escolham sempre. Para este estudo se desenvolveu uma metodologia quantitativa, que buscou descrever 
o comportamento destes enfoques nos operadores logísticos da cidade de Bogotá, identificando descobertas relevantes para este setor da capital colombiana.

Palavras-chave: Branding, valor de marca, operadores logísticos, internacionalização, serviços de operação logística.

\section{INTRODUCCIÓN}

La globalización es uno de los cambios más importantes que las compañías están viviendo hoy, y por tanto la entrada de las empresas colombianas a nuevos mercados se convierte en una de sus metas, pues quieren sobrevivir y obtener los mejores resultados en cuanto a la eficiencia e ingresos, la única posibilidad es expandirse al mundo (Restrepo y Rosero, 2002). La gestión de marca se ha convertido en uno de los temas obligados para los gobiernos, las organizaciones privadas y públicas, las ciudades y de los países (Martínez, 2007), por tal motivo se entiende que para internacionalizarse es necesario gestionar el crecimiento de la marca, ya que gracias a su planificación se pueden evidenciar logros importantes en el fortalecimiento de la competitividad.

Los operadores logísticos son una modalidad de contratación reciente en el país que viene posicionándose en el mercado gracias a la eficiencia de las operaciones logísticas, las economías de escala y la simplificación de las operaciones en la cadena de abastecimiento (González, 2015).
Por tanto, es importante analizar las estrategias de internacionalización de las empresas de servicios logísticos en Bogotá, partiendo de sus enfoques de brand equity y el éxito de las mismas, puesto que, bajo el enfoque estratégico, la internacionalización de empresas es el resultado de la adopción de una serie de estrategias en las que se consideran tanto los recursos y capacidades de la empresa, como las oportunidades y amenazas del entorno (Leandro, 2013).

Una empresa debe reconocer como factor determinante su marca, pues ella de manera diferencial e inimitable es la que crea, otorga y agrega valor a sus productos y/o servicios; de igual forma, como lo definen Kotler y Armstrong (2008), una marca se identifica con su nombre, término, signo, símbolo, diseño o también puede ser una combinación de todos estos elementos, que permiten identificar a un fabricante $\mathrm{o}$ al vendedor de un producto o servicio y diferenciarlo de la competencia.

Para lograr otorgarle un valor a la marca y posicionarla, es necesario que las empresas realicen una estrategia acertada dentro del proceso 
del branding. El brand equity puede ser una gran herramienta para cumplir con tal objetivo; según Aaker (1991) se entiende como un conjunto de activos y pasivos vinculados con la marca, con su nombre y su simbología, que incorporan o disminuyen valor suministrado por un producto o servicio intercambiado a los clientes de la compañía

Por otra parte, es notoria la importancia que tiene el brand equity en relación con la logística de las empresas, debido a que los clientes buscan satisfacer sus necesidades, mientras las empresas buscan fidelizar sus clientes, lo que se logra a través del ofrecimiento de un buen servicio. Por esta razón, es importante manejar una buena logística, para hacer llegar al cliente lo que necesita de manera oportuna, y es ahí cuando la marca y la calidad de la misma se relacionan directamente con la logística y sus procesos. Vallejo y Camacho (2010) hacen un aporte indicando "La marca no es una propiedad pero sí representa a una compañía. Entraña un significado que los consumidores consideran apropiado e identifican a la empresa con este significado, el cual va asociado a la experiencia personal del consumidor con el producto o servicio" (p. 3).

En la actualidad, muchas empresas comprenden la importancia del brand equity, reconociéndolo como material relevante en los cimientos de la marca; cuando una empresa implementa y gestiona modelos de valoración, puede fortalecer las finanzas de la empresa, obtener mayor capital de trabajo y aportar a una visión realista de los accionistas (Espósito \& Barbosa, 2001).

En el caso de la presente investigación, la implementación del brand equity en las empresas que ofrecen servicios de operación logística en Bogotá se hace más que necesaria, puesto que cuentan con una gran trayectoria en el mercado y sus servicios van ligados $100 \%$ a los procesos de aquellas compañías que los requieren. Así mismo, la relevancia de este sector en la economía del país es evidente, puesto que, según los estudios realizados por el Departamento de Planeación Nacional, en todo el país se encuentran aproximadamente un total de 264 empresas prestadoras de servicios logísticos, localizadas en su mayoría en Bogotá y Medellín, ciudades que cuentan con $50 \%$ de estas organizaciones (Departamento de Planeación Nacional, 2015).

Por otro lado, es importante identificar los sectores en donde tienen mayor acogida las empresas que tercerizan las operaciones de logística. Tal como se evidencia en la figura 1 
el sector de las manufacturas abarca del comercio mayorista y minorista el $51,3 \%$ de estas empresas, sien(Departamento de Planeación Nado el sector menos representativo el cional, 2015).

\section{Figura 1. Usuarios servicios logísticos}

" Sector Básico
"Sector Manufactura
- Sector Comercio
Mayorista y Minorista
- Proveedores de Servicios
No Logisticos

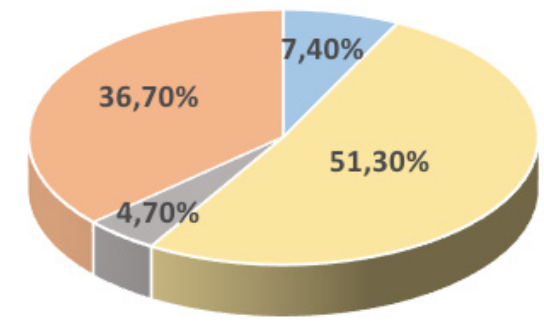

Fuente: Departamento de Planeación Nacional (2015).

Además, se estableció un estudio de los principales servicios que ofrecen los operadores logísticos y los que son más requeridos por las empresas que hacen uso de estos, siendo la ope- ración logística terrestre la de mayor participación con un 37,1\%, seguida de la operación logística marítima con un 18,9 \% (figura 2) (DepartamentodePlaneaciónNacional,2015).

Figura 2. Prestadores de servicios logísticos
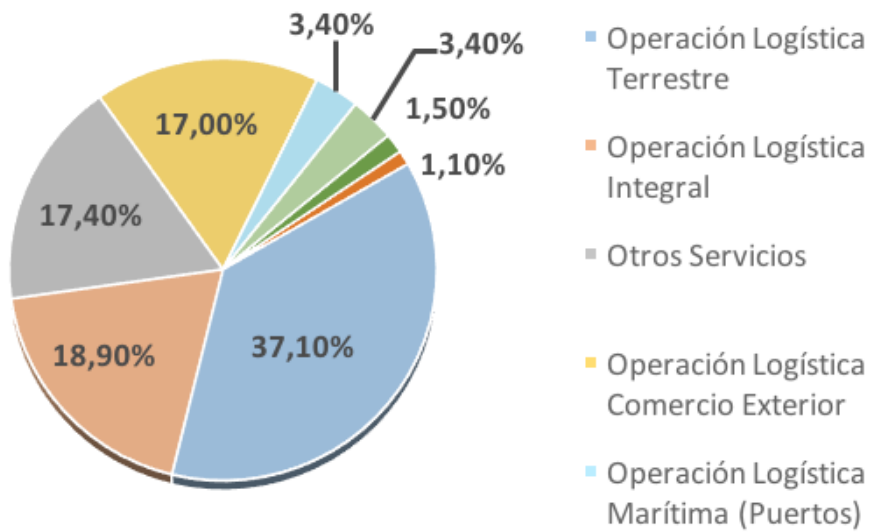

Fuente: Departamento de Planeación Nacional (2015). 
Son diversos los servicios prestados por los operadores logísticos a las empresas, lo cual permite que sus procesos no se vean afectados por contratar cada uno de ellos con proveedores diferentes, sino que todos sean ofrecidos por uno solo, lo que les permite reducir costos sino y, a la vez, ser más eficientes. El servicio con mayor requerimiento es el de transporte y distribución con un $37 \%$, y no menos importante pero con menor uso se encuentra la logística de reversa con un $7 \%$ (figura 3) (Departamento de Planeación Nacional, 2015).

\section{Figura 3. Servicios ofrecidos}

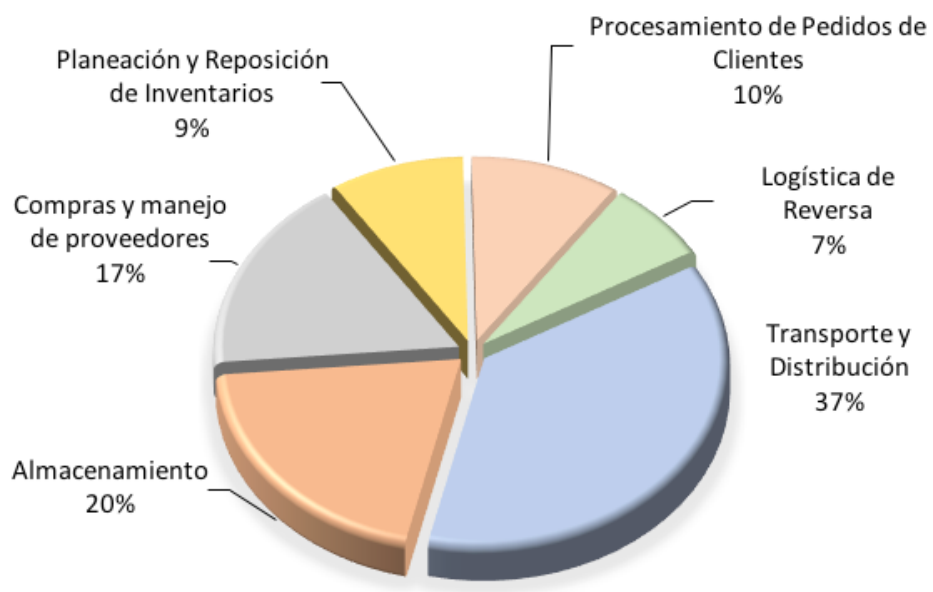

Fuente: Departamento de Planeación Nacional (2015).

De igual manera, es relevante identificar los porcentajes de eficiencia en los servicios prestados por los operadores logísticos, pues estos permiten que las compañías posicionen su marca en el mercado y logren mantenerla, mediante el reconocimiento de la calidad y el cumplimiento. Esto se se evidencia cuando el $92,2 \%$ de las entregas se realizan de manera completa, cuando las entregas no sólo son completas sino también a tiempo $(77,3 \%)$ y cuando de cada 10 pedidos 2 llegan tarde siendo $(83,8 \%$ ) (Departamento de Planeación Nacional, 2015).

Finalmente, se evalúan los indicadores de calidad, teniendo en cuenta el tamaño de las empresas que trabajan como operadores logísticos, siendo las grandes empresas las que obtienen los mayores porcentajes en estos indicadores de calidad, 
aunque no muy lejos de estas se encuentran las empresas pequeñas; tomando como ejemplo los pedidos sin daños, las grandes empresas cuentan con un $95,6 \%$ frente a un $92,6 \%$ de las empresas pequeñas (Departamento de Planeación Nacional, 2015).

Tabla 1. Indicadores de calidad por tipo de empresa

\begin{tabular}{|l|c|c|c|}
\cline { 2 - 4 } \multicolumn{1}{c|}{} & $\begin{array}{c}\text { Empresa } \\
\text { grande }\end{array}$ & $\begin{array}{c}\text { Empresa } \\
\text { mediana }\end{array}$ & $\begin{array}{c}\text { Empresa } \\
\text { pequeña }\end{array}$ \\
\cline { 2 - 4 } \multicolumn{1}{c|}{} & Promedio & Promedio & Promedio \\
\hline Pedidos sin error en documentos & $93,0 \%$ & $90,8 \%$ & $82,9 \%$ \\
\hline Pedidos sin daños & $95,6 \%$ & $93,6 \%$ & $92,6 \%$ \\
\hline Pedidos sin problemas de calidad & $91,0 \%$ & $92,3 \%$ & $87,5 \%$ \\
\hline Pedidos a tiempo y completos & $77,2 \%$ & $82,0 \%$ & $75,1 \%$ \\
\hline PEDIDO PERFECTO & $62,4 \%$ & $64,3 \%$ & $50,4 \%$ \\
\hline
\end{tabular}

Fuente: Departamento de Planeación Nacional, 2015

Frente a lo anterior, desde esta investigación surge como principal problemática analizar cuál es el comportamiento de los enfoques estratégicos del brand equity. Para ello se plantea como objetivo general caracterizar los enfoques estratégicos del brand equity en las empresas que internacionalizan servicios de operación logística en la ciudad de Bogotá. Como etapas fundamentales en la obtención de este objetivo se propone revisar en el estado del arte los enfoques estratégicos de brand equity que son más aplicados en el servicio de operación logística, explorar el manejo de dichos enfoques estratégicos en las empresas de servicios de operación logística, y reconocer su aplicación en las empresas de operación logística en la ciudad de Bogotá.

Con el fin de conceptualizar los elementos teóricos que enmarcan la investigación se presenta la siguiente revisión de la literatura.

\section{REVISIÓN DE LA LITERATURA}

\subsection{Enfoques estratégicos de brand equity}

Inicialmente, es importante conocer al detalle el concepto de marca. Esta puede ser un nombre, un símbolo y tiene como función identificar un bien o servicio, y diferenciarlo de los 
demás en el mercado o en un grupo determinado; por lo tanto se puede afirmar que si un vendedor o comerciante crea en un nuevo producto, un nombre o un símbolo, dicho comerciante estaría creando una marca (Keller, 2002). En apoyo a esta definición, Gil (2010) indica que la marca "ha sido entendida como un símbolo o palabra que identificaba un activo económico frente a la competencia" (p. 22). Se puede deducir que la marca es la identidad de un producto o servicio, y por tanto lo diferencia de los demás productos o servicios del mercado. Ahora bien, el reconocimiento que tienen las marcas en el mercado puede aumentar o disminuir dependiendo de las distintas características del mismo producto o servicio, ya sea calidad, precio etc.

Teniendo en cuenta lo anterior, la marca está ligada a los activos y responsabilidades de la misma, ya que si en algún momento una determinada marca se cambia, todo cambiará con ella. Según Ortegón (2013) la perspectiva financiera del valor de la marca es un factor importante para la misma; dicha perspectiva resalta el papel de la marca como un activo empresarial, que puede afectar positiva o negativamente los flujos de caja, el valor de las acciones y los precios de venta de las acciones, entre otros; por esto, la marca es un aspecto muy importante, ya que si está bien posicionada en el mercado, va a estar más presente en la mente de los consumidores, generando más consumo del producto o servicio en el mercado, lo que le permitirá obtener a la compañía más rentabilidad e ingresos. Sin embargo, para lograr que esta tenga un buen posicionamiento, es necesario tener en cuenta la opinión de los consumidores para el crecimiento de la marca.

Según Torres (2013), "los consumidores plasman la imagen de la marca de un determinado producto o servicio en su mente a través de las diferentes actividades del marketing, estas pueden ser la experiencia directa, información de terceras personas o mediante supuestos a través de los cuales crean sus propias conclusiones sobre la marca" (p. 43).

Buil, De Chernatony y Martínez (2013) también indican la importancia de los consumidores en el éxito que tenga la marca, es por esta razón que deben ser tenidos en cuenta para desarrollar propuestas de medición, que valoren sus percepciones, preferencias y comportamientos, pues son ellos quienes califican la marca y transmitir una buena o mala imagen de la misma entre sus conocidos. 
Consecuentemente, aparece un concepto que es el branding; para Siabato (2014) "la integración de estrategias de branding busca construir una marca fuerte con un valor significativo debido a que esto se traduce en beneficios para las compañías" (p. 25). Para una empresa es importante conocer la percepción de sus clientes en relación con su marca y mantenerla viva en el mercado; según Crescitelli y Mandakovic (2016), la marca es el objetivo final en el que se busca concentrar y materializar todos los esfuerzos del proceso de la gestión de la misma.

Para Aaker (1991) brand equity es una colección de los activos de la marca y de sus responsabilidades; el nombre y símbolo de una marca puede aumentar o disminuir el valor provisto por un producto o servicio, y cuando cambian pueden cambiar a su vez el activo de la marca y sus responsabilidades.

Por su parte, Forero (2014) plantea que el brand equity permite a las empresas conocer de manera más clara y precisa la percepción que tie- nen sus clientes acerca de la marca. Esto permite contar con la información verídica a la hora de tomar decisiones y poder mantener el nombre de la marca en el tiempo; además, el Brand Equity permite conocer el reconocimiento que obtiene la marca y la importancia que tiene la misma en el mercado, aspecto que se evidencia en la vida cotidiana como consumidor, ya que, casi siempre, a la hora de adquirir un producto los consumidores ya tienen una marca presente, que recuerdan por la calidad del producto y por su precio, entre otros.

Para poder contar con la información suficiente y diseñar nuevas estrategias de marketing, Torres (2013) indica que el brand equity maneja distintos aspectos del cliente, como la recordación de la marca en la mente de los clientes y las características del producto que lo hacen más atractivo para los consumidores.

Para el desarrollo del brand equity hay que tener en cuenta que existen varios grupos de métodos o enfoques para aplicar (tabla 2). 
Tabla 2. Enfoques del brand equity

\begin{tabular}{|c|c|}
\hline Dimensiones & Ítems \\
\hline \multirow{4}{*}{ Rendimiento } & De esta marca de ... espero un rendimiento superior. \\
\hline & Es muy poco probable que esta marca de... ocasione imprevistos durante su uso. \\
\hline & Esta marca de... resulta fácil de usar. \\
\hline & Esta marca funcionará muy bien. \\
\hline \multirow{4}{*}{ Imagen Social } & Esta marca de... se adecua a mi personalidad. \\
\hline & Me siento orgulloso de poseer esta marca de... \\
\hline & Esta marca de... goza de gran aceptación entre mis amigos. \\
\hline & Podría decir que el estilo de la marca... se corresponde con mi propia personalidad. \\
\hline \multirow{2}{*}{ Valor } & Esta marca tiene un precio adecuado. \\
\hline & Considero que la marca me aporta un valor superior al precio pagado por ella. \\
\hline \multirow{3}{*}{ Confianza } & Confío en la empresa y en las personas que fabrican esta marca. \\
\hline & $\begin{array}{l}\text { Creo que esta marca se preocupa por satisfacer los gustos y necesidades de los } \\
\text { consumidores. }\end{array}$ \\
\hline & Pienso que esta marca no se aprovecha de los consumidores. \\
\hline \multirow{3}{*}{ Compromiso } & Siento un cierto cariño hacia esta marca de... \\
\hline & En general mis opiniones hacia esta marca de... son positivas \\
\hline & Con el tiempo, es muy probable que sienta un especial afecto por esta marca. \\
\hline
\end{tabular}

Fuente: Lassar, Mittal y Sharma, 1995.

Al realizar una comparación entre los diferentes tipos de medida del valor de marca expuestos por Villarejo (2002) es posible distinguir entre las medidas de índole intrínseca y extrínseca. Las primeras muestran en lo que la marca es fuerte con relación a la captación del consumidor, su predilección y la conducta mani- festada hacia la marca, mientras que las segundas demuestran la fortaleza de las marcas con relación a su sensibilidad ante los cambios en las políticas de marketing de la empresa y de su competencia. Se considera entonces, que las medidas de tipo intrínseco son apropiadas para la medición del valor de marca. 
Tabla 3. Medidas intrínsecas del valor de marca

\begin{tabular}{|l|l|}
\hline \multicolumn{1}{|c|}{ Dimensiones } & \multicolumn{1}{c|}{ Ítems } \\
\hline Conocimiento & Primera marca mencionada y reconocimiento de la marca. \\
\hline \multirow{2}{*}{ Actitud hacia la marca } & Escala de 10 puntos sobre la calidad percibida de la marca. \\
\cline { 2 - 3 } Preferencia & Porcentaje de consumidores con intención de comprar la marca. \\
\hline \multirow{3}{*}{ Datos de elección } & $\begin{array}{l}\text { Utilidad proporcionada por la marca mediante análisis conjunto. Preferencia } \\
\text { Prima de precio relativa (en comparación al precio actual) que iguala la utilidad } \\
\text { de la marca con la proporcionada por una marca "media" para la categoría de } \\
\text { producto. }\end{array}$ \\
\cline { 2 - 3 } & $\begin{array}{l}\text { Elección de la marca para un nivel de precios inferior al precio de mercado. } \\
\text { Proporción de personas que usan principalmente o de manera exclusiva la } \\
\text { Parca. } \\
\text { Porcentaje de personas que han probado la marca. }\end{array}$ \\
\hline
\end{tabular}

Fuente: Villarejo, 2002.

\subsection{Internacionalización}

La internacionalización es uno de los enfoques de este artículo y se debe partir de la importancia del mismo, entendiendo que es un proceso llevado a cabo por las empresas para entrar a los mercados internacionales y asumiendo que las operaciones a gran escala son un requerimiento para las empresas multinacionales, donde los procesos de internacionalización tardan más tiempo en realizarse (Barbosa, 2015).

Si se mira desde un enfoque estratégico, la internacionalización de las empresas es el resultado de la adopción de una serie de estrategias en las que se consideran tanto los recursos y capacidades de la empresa, como las oportunidades y amenazas del entorno. Además, se tiene que entender que no basta con concentrar actividades, sino que resulta necesario desarrollar una capacidad de adaptación a cada país y mercado; no se puede vender el mismo tipo de productos en Costa Rica que en Alemania (Leandro, 2013).

No cabe duda de que el proceso de internacionalización de la economía, unido al tejido empresarial es un eslabón más de la globalización, por lo que no es posible hablar de internacionalización dejando de lado el fenómeno de globalización (Gómez, 2010). En Colombia, la internacionalización de las empresas es un tema con grandes obstáculos, entre ellos la falta de conocimiento 
del mercado y la ausencia de experiencia. De acuerdo con la posición presentada por Tabares, Anzo y Estrada (2014), estos obstáculos son reducidos a través del aprendizaje y la toma de decisiones graduales en el mercado internacional, pero actualmente este panorama se vuelve más positivo ya que cada vez son más las empresas que desarrollan sus operaciones en el mercado internacional, e incluso muchas de ellas las inician cuando aún son pequeñas y no cuentan con reconocimiento suficiente en el mercado doméstico.

El objetivo de una empresa multinacional es la reproducción casi exacta de la empresa matriz en cada una de las filiales en el extranjero. Según Leandro (2013), para este proceso hay unos pasos claves: primero, la empresa se desarrolla en su mercado nacional; segundo, se comienza a llevar a cabo exportaciones irregulares; tercero, se emplean agentes independientes; cuarto, se implantan filiales comerciales, y finalmente, para poder contar con la información suficiente y diseñar nuevas estrategias de marketing, se ponen en marcha las filiales productivas.

También es de gran importancia entender que los gobiernos tienen una gran responsabilidad en crear las condiciones que favorezcan el desarrollo de las empresas, por lo que deben impulsar la creación de sectores avanzados y estimular la demanda, las relaciones entre empresas y la rivalidad entre éstas. Entendiendo que, los países han llegado a un momento de integración en donde sus actores políticos, económicos y sociales se enfrentan a un cambio radical, la producción de bienes ha quedado en un segundo plano para dar paso a los servicios, el conocimiento y la innovación (Vargas \& Hernández, 2006).

Según Reina (2010), para que se dé una buena internacionalización se tiene que tener en cuenta el acceso a los mercados; esta es, justamente, la dimensión en la que el país más ha avanzado en los últimos años, de las tres áreas fundamentales para avanzar en la internacionalización de la economía, pero es evidente el rezago frente a lo que han hecho otros países de la región en la negociación de acuerdos de libre comercio.

El Gobierno tiene a disposición diferentes herramientas mediante entidades públicas o mixtas que le permitirán mejorar sus procesos empresariales con miras a una apertura internacional, y cuenta con programas no son solo de capacitación, sino también de mecanismos para el fortalecimiento empresarial, como los que ofrece la Agencia Pública de Empleo y Propaís; además, cuenta 
con las normativas de la Superintendencia de Industria y Comercio, la Dirección Nacional de Derechos de Autor y Colombia Compra Eficiente (Procolombia, 2017).

También debe trabajar duramente y de manera simultáneaen tres áreas de gran enfoque para poder llegar al avance que se busca y lograr así una inserción efectiva de Colombia en la economía internacional. La primera es en la búsqueda de un mayor acceso a más mercados, la segunda es la eliminación del sesgo antiexportador y la última es la superación de obstáculos básicos a la competitividad de la economía colombiana. Si no se tienen en cuenta todas las áreas, el progreso que se logre en una de ellas se verá frustrado por el atraso de las otras dos y no se conseguirá una mayor inserción global de la economía (Reina, 2010).

Como una muestra de lo anteriormente indicado, el DANE (2015) indica la composición de exportaciones de servicios de 2015, en una figura en la que se puede notar que el servicio con mayor porcentaje es el de viajes con un $55 \%$, seguido de transporte, con un $21 \%$ (figura 4).

\section{Figura 4. Composición exportaciones de servicios}
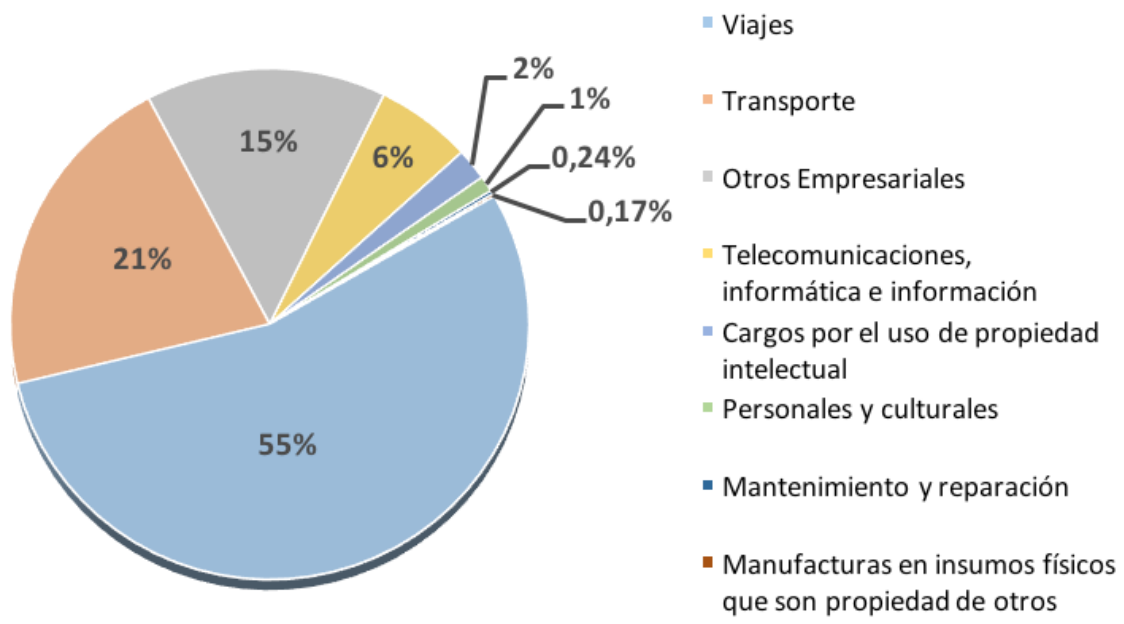

Fuente: DANE, cálculos de la revista Dinero (2015). 


\subsection{Operadores logísticos}

La logística como tal es de enorme importancia en sí misma, más aún cuando genera un apoyo en el crecimiento del sector privado y en los demás factores de la economía de un país o de una región. Un proceso de logística eficiente y accesible a todos los mercados representa un elemento clave para que las compañías, en general, y en el caso particular, las pymes, tengan la capacidad de llegar a competir con éxito en el nuevo marco global (Kirby \& Brosa, 2011).

Los servicios de logística integral permiten dar un valor agregado a los productos, bienes o servicios en los cuales son utilizados; en un concepto internacional, Vallejo, Cortés, y Olaya (2010) indican que "La logística se ha interpretado en el contexto internacional como una actividad que añade valor a los productos o servicios para la satisfacción del cliente" (p. 170).

Los operadores logísticos ofrecen diversas actividades en la cadena de suministro, como son el transporte, el almacenamiento, la distribución y la gestión de inventario, entre otras, haciendo uso de sistemas de información con el fin de ser más eficientes en dichas operaciones, para presentar a sus clientes la solución logística que se adecue a sus necesidades (Osorio, 2015).
De igual forma, son compañías que ofrecen de manera integral servicios de logística en el comercio exterior para empresas de los diferentes sectores de la economía; según Tompkins Associates (s.f.), estas empresas prestan servicios integrales de logística en la cadena de abastecimiento y la mayoría de ellos operan la totalidad de la logística de aquellas empresas que los contratan.

Es importante reconocer los beneficios que trae la tercerización de estos servicios. Siguiendo lo expuesto por Mora (2015), dentro de los principales beneficios están reducir los gastos de operación, liberar recurso humano y contar con mayores fondos de capital, con el propósito de invertir en el objeto social de la empresa y contar con proveedores de clase mundial y que cuentan con procedimientos mejor estructurados.

Para Colombia, los servicios de los operadores logísticos son una reciente forma de contratación que se ha posicionado en los diferentes mercados gracias a la calidad, eficiencia y garantía de cada uno de los procesos que acompañan, permitiendo a las empresas que contratan estos servicios la simplificación de las operaciones en su cadena de abastecimiento, los cambios en costos fijos y la liberación de su recurso 
humano para destinarlo totalmente al objeto principal de la empresa (Vallejo, Cortés \& Olaya, 2010).

Según Santos (2015), en este momento el país y el sector de servicios de logística se encuentran en un momento importante, por lo que es necesario romper algunos paradigmas y así poder mejorar productivamente, y de forma complementaria y competitiva.
En la figura 5 se muestra el crecimiento de Colombia en el índice de desempeño logístico entre 2014 y 2016, en donde es posible establecer que no ha sido el más significativo; esto debería ser tomado en cuenta por las empresas que prestan estos servicios, para realizar una mejora en cada proceso, para lograr posicionar su marca en el mercado y presentar una ventaja competitiva ante sus clientes.

Figura 5. Colombia - Índice desempeño logístico

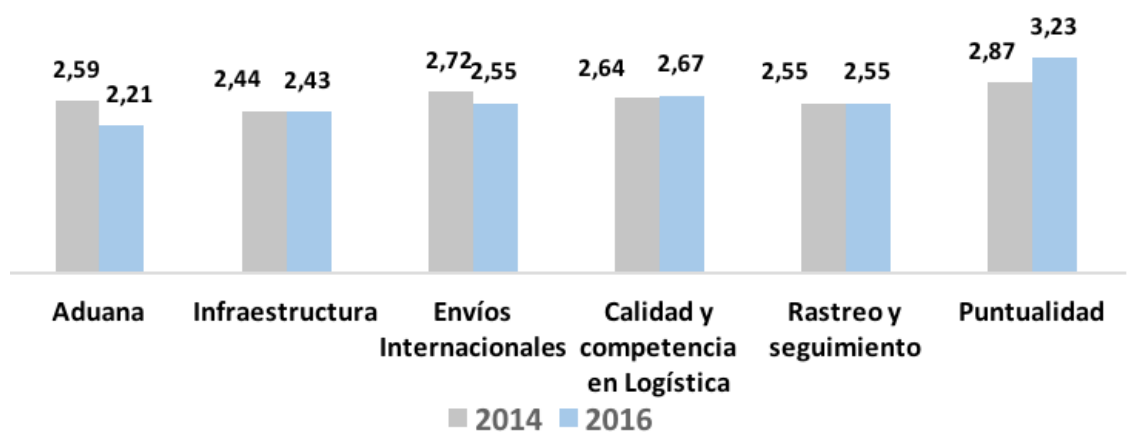

Fuente: Analdex (2016).

Posteriormente, se compara a Colombia frente a 160 países en su índice de desempeño logístico (figura 6). Allí se puede ver que el país ha mejorado en ciertos aspectos, en relación a los resultados obtenidos para 2014, como lo fue en puntualidad, rastreo, seguimiento y los servicios logísticos. Es evidente que, a pesar de los esfuerzos de las empresas por elevar estas cifras, lo relacionado con la infraestructura y los múltiples requisitos aduaneros dificultaron que dichos procesos llegaran ser más eficientes para los operadores logísticos y por ende para Colombia (Analdex, 2016). 
Figura 6. Colombia - Índice desempeño logístico (entre 160 países)

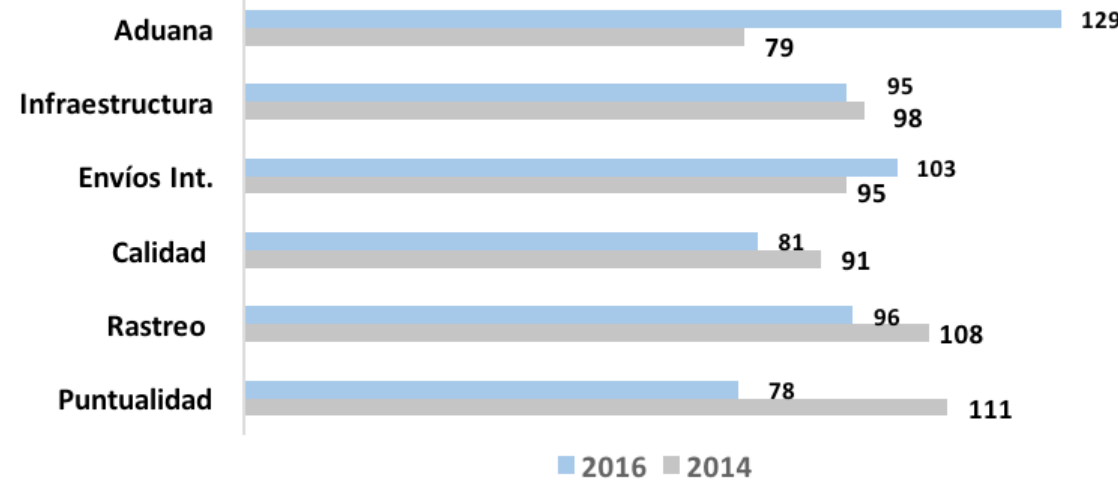

Fuente: Analdex (2016).

En una publicación realizada por el Sistema Nacional de Competitividad, Ciencia, Tecnología e Innovación, dentro del índice de desempeño logístico, Colombia ocupa la posición 94 entre 160 países, con un puntaje de 2,61, frente a un puntaje de 4,23 de Alemania, que ocupa el primer puesto (SNCCTI, 2016).

Así, a pesar de que las estadísticas no muestran a Colombia con puntajes más óptimos a nivel mundial, si es posible asegurar, tal como lo indica el Centro de Documentación para la Innovación de la Cadena de Suministro (2012), que la infraestructura y la experiencia de los operadores logísticos en el país permiten generar confianza al momento de tercerizar servicios logísticos.

\section{METODOLOGÍA}

De acuerdo al problema planteado para el presente estudio, se trabajó desde el enfoque metodológico cuantitativo que, según Hernández, Fernández y Baptista (2003) “utiliza la recolección y el análisis de datos para contestar preguntas de investigación y probar hipótesis establecidas previamente, y confía en la medición numérica, el conteo y frecuentemente en el uso de la estadística para establecer con exactitud patrones de comportamiento en una población" (p. 12).

Como unidad de información, la muestra se realizó con empresas de Servicios Logísticos que desarrollan procesos de internacionali- 
zación en la ciudad de Bogotá, las cuales fueron obtenidas de la base de datos de Procolombia, en donde actualmente existen 90 empresas que cumplieron con dicha característica. Para este estudio se estimó una muestra de 39 empresas con un nivel de confianza del $90 \%$ y un margen de error del $10 \%$ (tabla 4).

Tabla 4. Determinación de la muestra

\begin{tabular}{|c|c|}
\hline \multicolumn{2}{|c|}{ Tamaño de la muestra (población finita) } \\
\hline $\mathrm{N}$ & 90 \\
\hline \multirow{2}{*}{$\mathrm{Z}$} & $90 \%$ \\
\cline { 2 - 2 } & 1,65 \\
\hline $\mathrm{E}$ & $10 \%$ \\
\hline $\mathrm{p}$ & $50 \%$ \\
\hline $\mathrm{q}$ & $50 \%$ \\
\hline $\mathrm{n}$ & 39 \\
\hline
\end{tabular}

Fuente: elaboración propia.

El método utilizado para la selección de la muestra fue el muestreo probabilístico aleatorio simple, en el que se selecciona en donde cada uno de los elementos o personas de la población cuenten con las mismas posibilidades de ser incluido (Lombana, 2010).
El instrumento para la recolección de la información se propone desde las variables propuestas por Lassar, Mittal y Sharma (1995) y Villarejo (2002), las cuales fueron tomadas de las tablas 2 y 3 (tabla 5).

Tabla 5. Variables para la reconstrucción del instrumento

\begin{tabular}{|c|c|}
\hline \multicolumn{2}{|c|}{ Dimensiones } \\
\hline Rendimiento & Conocimiento \\
\hline Imagen Social & Actitud hacia la marca \\
\hline Valor & Preferencia \\
\hline Confianza & Datos de elección \\
\hline \multicolumn{2}{|c|}{ Compromiso } \\
\hline
\end{tabular}

Fuente: elaboración propia a partir de Lassar, Mittal y Sharma (1995), y Villarejo (2002).

\section{RESULTADOS}

De acuerdo con lo propuesto en la metodología, a continuación se presenta un análisis estadístico del comportamiento de los enfoques estratégicos del brand equity en el sector de las empresas que se seleccionaron como muestra (figura 7); frente a ello se obtiene lo siguiente: 
Figura 7. Tipo de operación logística

Fuente: elaboración propia.

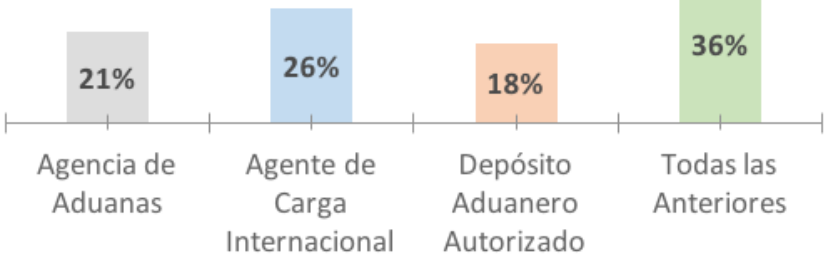

De las empresas encuestadas, el puede manejar toda su operación $36 \%$ ofrece todos los servicios lo- con una sola empresa. Según lo exgísticos tenidos en cuenta, seguido puesto por Santos (2015), el país y con el $26 \%$ para los servicios de el sector de servicios de logística agente de carga internacional, don- se encuentran en un momento imde se ve reflejado que en Bogotá portante, por lo que es necesario son de mayor porcentaje las empre- romper algunos paradigmas para sas que ofrecen todos los servicios mejorar productivamente y de forlogísticos, puesto que el cliente ma competitiva.

Figura 8. Tamaño de la empresa

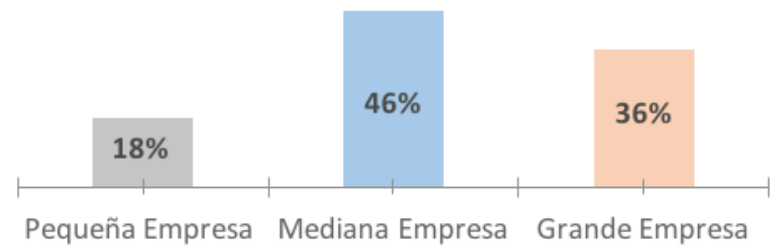

Fuente: elaboración propia.

El $46 \%$ de las empresas encuestamayoría de empresas están en un das son de un tamaño mediano, seguido del $36 \%$ de grandes empresas, proceso de crecimiento y competilo cual demuestra que en Bogotá la tividad (figura 8). 
Figura 9. Imagen social de la marca

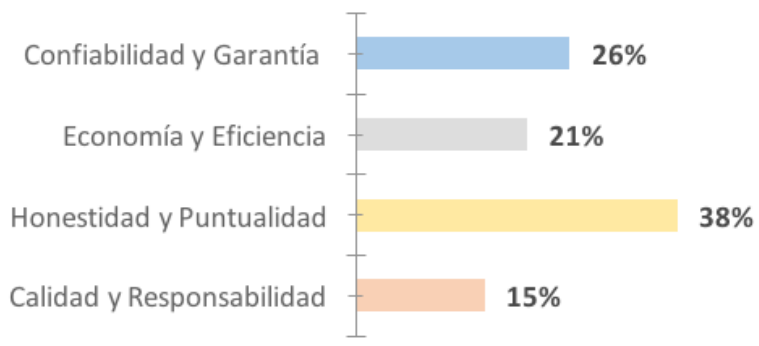

Fuente: elaboración propia.

Se evaluó la imagen social de la marca como un enfoque del brand equity, donde el $38 \%$ indica que los valores que refleja la empresa hacia la imagen social son la honestidad y la puntualidad, seguidos del $26 \%$, con confiabilidad y garantía (figura 9); este resultado concuerda con lo propuesto por Lassar, Mittal y Sharma (1995), quienes describen que "esta marca se adecua a mi personalidad, Me siento orgulloso de poseer esta marca, esta marca goza de gran aceptación entre mis amigos, esta marca corresponde con mi propia personalidad" (p. 23).

Figura 10. Relación del valor frente al precio de los productos

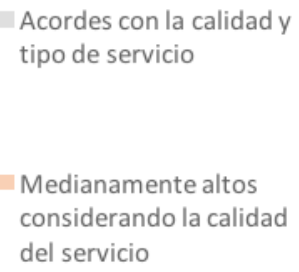

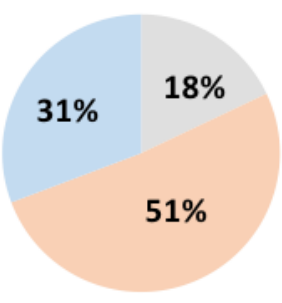

Fuente: elaboración propia.

El anterior indicador se interpreta del siguiente modo: $51 \%$ medianamente alto, considerando la calidad del servicio; $31 \%$ alto, garantizando tiempos, responsabilidad y eficiencia en cada servicio, y $18 \%$, acordes a la calidad y el tipo de servicio (figura 10). Para Kotler y Armstrong (2008), el valor determinante de una empresa debe ser su marca, en el entendido de que ella es diferencial e inimitable ya que crea, agrega y otorga valor a sus productos y/o servicios. 
Figura 11. Nivel de confianza frente a las marcas

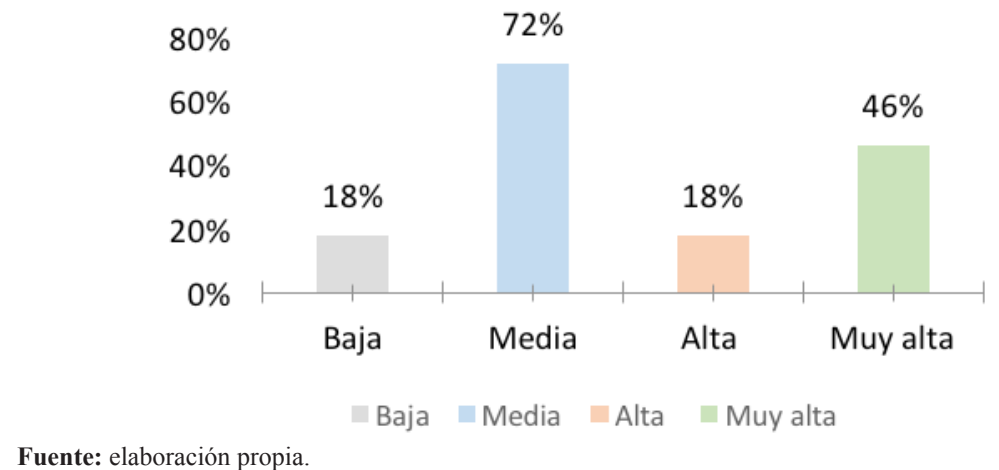

Se midió el nivel de confianza que como "confío en la empresa y en las tienen las empresas frente a las mar- personas que fabrican esta marca, cas como una estrategia del brand creo que esta marca se preocupa por equity, mostrando un $72 \%$ de con- satisfacer los gustos y necesidades fianza media, seguido de $46 \%$ de de los consumidores, pienso Que muy alta (figura 11), lo cual muestra esta marca no se aprovecha de los un alto porcentaje la confianza que consumidores. Dando un apego a refleja la empresa como marca. Se la marca y a lo que refleja hacia los evalúa esta confianza en la marca clientes" (p. 23). según Lassar, Mittaly Sharma(1995)

Figura 12. Nivel de recordación de marca por parte de los clientes

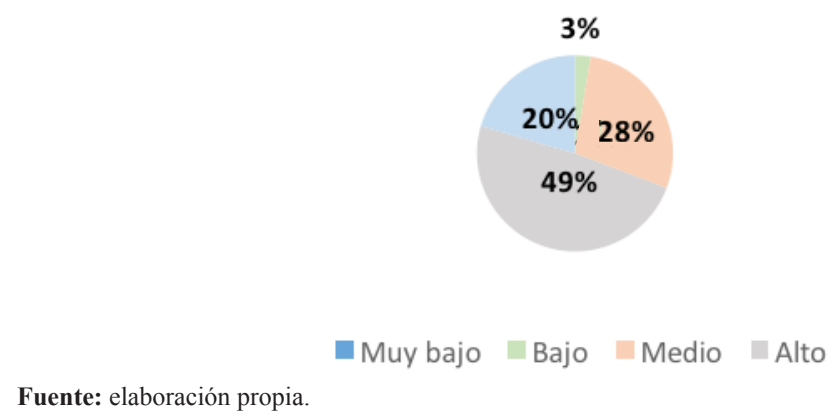


En el resultado anterior se puede ver el nivel de recordación de la marca por parte de los clientes: $49 \%$ alto, $28 \%$ medio, $21 \%$ muy alto, $3 \%$ bajo y $0 \%$ muy bajo (figura 12 ). De acuerdo con Torres (2013), se indica que el brand equity utiliza diferentes aspectos de los clientes, para poder tener la información suficiente y lograr diseñar nuevas estrategias de marketing, como, por ejemplo, mantener en la mente de los clientes la marca y sus características en cuanto a aquellos productos más atractivos.

Figura 13. Valores que representan la marca para los clientes

Calidad
Puntualidad
Economia
Eficiencia
Permanencia

Fuente: elaboración propia.

Otro punto a evaluar son los valores que representan a la marca, aquellos que se reflejan a los clientes, donde se tuvo en cuenta la calidad, puntualidad, economía, eficiencia y permanencia. Se obtuvo como resultado: $36 \%$ puntualidad, $23 \%$ economía, $23 \%$ permanencia, $10 \%$ calidad y $8 \%$ eficiencia (figura 13). Según lo expuesto por Villarejo (2002) es posible distinguir entre las medidas de índole intrínseca y extrínseca. Las primeras muestran en lo que la marca es fuerte con relación a la captación del consumidor, su predilección y la conducta manifestada hacia la marca, mientras que las medidas extrínsecas demuestran la fortaleza de las marcas con relación a su sensibilidad ante los cambios en las políticas de marketing de la empresa y de su competencia. Se considera entonces que las medidas de tipo intrínseco son apropiadas para la medición del valor de marca.

\section{CONCLUSIONES}

La investigación desarrollada permite evidenciar la importancia del branding en el posicionamiento de una marca, sobre todo cuando está siendo calificada desde la percepción de su calidad global e integral por parte del consumidor, es decir, conocido como brand equity, lo cual fue 
analizado en las diferentes empresas que internacionalizan sus servicios de operación logística en Bogotá.

Como objetivo principal se buscaba caracterizar los enfoques estratégicos del brand equity en dichas empresas, en donde, mediante el análisis de las encuestas realizadas, fue posible establecer que estas variables, pasando desde su rendimiento, imagen social, valor, confianza y compromiso, entre otras, muestran la fortaleza de las marcas para estas empresas de servicios logísticos, frente al mercado y frente a la competencia, lo que les permite obtener un posicionamiento importante $\mathrm{y}$ único, generando una constante recordación de la marca.

Los resultados arrojados por el estudio aportan conocimiento, a la vez que son consecuentes con la literatura presentada a lo largo de esta investigación, puesto que permiten evidenciar la importancia de la correcta administración sobre las estrategias que implementen las empresas para lograr dar un valor a su marca y por ende a sus productos o servicios, además de generar empatía y fidelización en los clientes.

El método aplicado para llevar a cabo esta investigación permitió analizar el valor de la marca desde una perspectiva interna de las empresas de cómo sus clientes han calificado los servicios que ofrecen al mercado y cómo se ven frente a su competencia, lo que les permite evaluar y evidenciar falencias en las políticas que han establecido e implementado para el posicionamiento de su marca.

Así mismo, los resultados de la investigación realizada para el presente estudio permitieron identificar que estas empresas de servicios logísticos reconocen que sus clientes toman la decisión de trabajar con ellos, no sólo teniendo en cuenta el precio de los servicios que presentan, sino también el valor agregado que se les otorga; caso puntual como se logró establecer en la figura 9, en donde cualidades de estas empresas, como honestidad y puntualidad, toman mayor importancia comparadas con otras, como la calidad y la responsabilidad, que aunque son igual de importantes que las anteriores, obtienen menor porcentaje, pues hoy en día cualidades como la puntualidad son más requeridas, pues permiten la reducción en costos y ofrecen a los clientes de estas empresas un mayor nivel de competitividad.

Por otro parte, el nivel de recordación que tienen los clientes hacia la marca de cada una de estas empresas, se ve directamente relacionado 
con el nivel de confianza que se brinda en cada uno de los servicios; a pesar de que en los resultados obtenidos para esta variable el mayor nivel de confianza fue de nivel medio, es un punto que debe ser tomado como oportunidad, de manera tal que al momento en que una de estas empresas busque tener un punto relevante en diferenciación con su competencia, pueda trabajarlo exigentemente y así llegar a abarcar la mayor parte del mercado, sólo aplicando correctamente estrategias dirigidas a esta variable.

De igual manera, el objeto de estudio del presente artículo le permitirá identificar a otras empresas con estas mismas características de mercado, o aquellas que se encuentren en proceso de llegar a serlo cuáles son aquellas variables en las que pueden llegar a marcar diferencia, ser fuertes y de manera particularmente sobresaliente ocupar los mayores porcentajes de participación en el sector del comercio exterior, lo que no sólo les permitiría trabajar a nivel nacional, sino llegar a ser reconocidas a nivel internacional.

Así, se puede concluir que las empresas que internacionalizan servicios logísticos en la ciudad de Bogotá, y que fueron partícipes del presente estudio, pueden llegar a establecer procesos de mejora y acondicionar sus estrategias de posicionamiento de marca, con el fin no sólo de obtener un gran reconocimiento por parte de sus clientes y de su misma competencia, sino también de generar un valor económico y representativo para su propia empresa.

\section{REFERENCIAS}

Aaker, D. (1991). Managing Brand Equity. Recuperado de http:// www.inovaconsulting.com.br/ wp-content/uploads/2016/09/ managing-brand-equity-by-david-aaker.pdf

Analdex (2016). Índice de Desempeño Logístico 2016. Recuperado de http://www.analdex.org/wpcontent/uploads/2016/02/201607-11-ndice-de-Desempeo-Logstico-2016-Banco-Mundial.pdf

Barbosa, D., Ayala, A., \& López, C. (2015). Dinámica exportadora de las empresas born global en Colombia. Revista Finanzas y Politica Económica, 5(1), 121-150.

Buil, I., De Chernatony, L., \& Martínez, E. (2013). La importancia de medir el valor de marca desde la perspectiva del consumidor: Evidencia empírica en España y el Reino Unido. Revista de Ciencias Sociales, 19(2), 225-237.

Centro de Documentación para la Innovación de la Cadena de Suministro. (2012). Tercerizar o no tercerizar: he ahi la cuestión. 
Recuperado de http://innovasupplychain.pe/articulos/3112tercerizar-o-no-tercerizar-heahi-la-cuestion

Composición de exportaciones de servicios 2015 (13 de octubre de 2015). Revista Dinero. Recuperado de http://www. dinero.com/economia/articulo/ exportaciones-servicios-colombia/214572

Crescitelli, E., \& Mandakovic, T. (2016). El patrimonio de marca (Brand Equity) en el sector de la educación: un estudio exploratorio en el segmento de cursos de MBA. Fórum Empresarial, 15(1), 45-63.

Departamento de Planeación Nacional.(2015).Colombiaes Logística. Encuesta Nacional de Logística 2015. Recuperado de http://www. colombiacompetitiva.gov.co/ prensa/2015/Paginas/Colombiaes-Logistica-La-Encuesta-Nacional-de-Logistica-2015.aspx

Espósito, I., \& Barbosa, L. (2001). Brand Equity: Modelos de Valuación. Recuperado de http:// www.ucema.edu.ar/posgradodownload/tesinas2001/Esposito-MADE.pdf

Forero Siabato, M. (2014). Determinación de la aplicabilidad del Brand Equity basado en el consumidor para el contexto colombiano (tesis de doctorado), Universidad Nacional de Colombia, Bogotá, Colombia.
Garnica, A. (2002). Brand Equity: El valor de marca. Recuperado de http://cvonline.uaeh.edu.mx/ Cursos/Lic virt/Mercadotecnia/DMKT011/UNIDAD $\% 20$ 3/35 lec_Brand\%20Equity_valor de la marca.docx

Gil, J. (2010). Branding. Tendencias y retos en la comunicación de marca Vol. 38. Catalynia: Editorial UOC.

González, J. (2015). Contratación logística en Colombia: implementación de un operador logístico integral. Semestre Económico, 18(38), 215-237.

Rave, E. (2010). La internacionalización de las empresas. Recuperado de https://www.ceipa.edu. co/lupa/index.php/lupa/article/ view/106/206

Hernández, R., Fernández, C., \& Baptista, P. (2003). Metodología de la investigación. La Habana: Editorial Félix Varela.

Keller, K. (2002). Branding and Brand Equity. Handbook of Marketing, Chennai, India: SAGE Publications Ltd.

Kirby, C., \& Brosa, N. (2011). La logística como factor de competitividad de las Pymes en las Américas. Recuperado de https://publications.iadb.org/ bitstream/handle/11319/5089/ La\%20log\%C3\%ADstica\%20 como $\% 20$ factor $\% 20$ de $\% 20$ competitividad $\% 20 \mathrm{de} \% 20$ 1 as $\% 20$ Pymes $\% 20$ en $\% 20$ 
1as $\% 20 \mathrm{Am} \% \mathrm{C} 3 \% \mathrm{~A} 9$ ricas. pdf? sequence $=1 \&$ isAllowed $=y$

Kotler, P., \& Armstrong, G. (2008). Fundamentos de marketing ( $8^{\mathrm{a}}$ ed.). New Jersey: Pearson-Prentice Hall.

Lassar, W., Mittal, B., \& Sharma, A. (1995). Measuring Customer-based Brand Equity. Journal of Consumer Marketing, 12(4), 11-19.

Leandro, A. (2013). El proceso de internacionalización de empresas. Tec Empresarial, 3(3), 18-25.

Martínez, S. (2007). Sistema de gestión comunicacional para la construcción de una marca ciudad o marca país. Signo y pensamiento, 26(51), 80-97.

Mora, L. (2015) Los operadores logísticos en Colombia. Catálogo de Logística. Recuperado de http:// www.catalogodelogistica.com/ temas/Los-operadores-logisticosen-Colombia +104324 ? pagina $=2$

Ortegón Cortázar, L. (2013). Relación entre valor de marca y las ventas. Un estudio aplicado en compañías agroquímicas Correlación entre valor de marca y valor de ventas. Revista Ciencias Estratégicas, (29), 104-124.

Osorio, H. (2015). Operadores 1ogísticos de cuarto nivel un tema para profundizar en Colombia. Zona Logistica, 15(82), 11-15.

Procolombia. (2016). Directorio de Distribución Física Internacional. Recuperado de http://www. colombiatrade.com.co/herra- mientas/dfi-directorio-de-distribucion-fisica-internacional

Santos, A. (2015). Perfil logístico de Colombia; Una visión hacia el mejoramiento estratégico de las operaciones nacionales e internacionales. Recuperado de http://www.urosario. edu.co/getattachment/Administracion/ur/Investigacion 1/ ur/Grupo-de-Investigacion/ Proyectos/4-Perfil-logistico-deColombia-una-vision-hacia-elmejoramiento-estrategico.pdf

Restrepo, M., \& Rosero, X. (2002). Teoría institucional y proceso de internacionalización de las empresas colombianas. Estudios Gerenciales, 18(84), 103-123.

Reina, M. (2010). Internacionalización de la economía colombiana: comercio e inversión. Bogotá: CAF, BID.

Sistema Nacional de Competitividad, Ciencia, Tecnología e Innovación. (2016). Índice de Desempeño logístico del Banco Mundial. Recuperado de http:// www.colombiacompetitiva.gov. co/prensa/2016/Paginas/Indicede-Desempeno-logistico-delBanco-Mundial.aspx

Tabares, S., Anzo, E., \& Estrada, L. (2014). La cooperación internacional en la internacionalización de pequeñas y medianas empresas de Medellín: un estudio de caso comparado. Estudios Gerenciales, 30(132), 314-324. 
Tompkins Associates (s.f.). Third Party Logistics. Recuperado de https://www.tompkinsinc.com/ en-us/Industries/Third-PartyLogistics

Torres Valverde, E., \& Padilla Rivadeneira, G. (2013). Medición de la intención de compra con base en un modelo de regresión logística de productos de consumo masivo (tesis de pregrado) Universidad de Chile, Santiago de Chile.

Vallejo, B. Cortés, E., \& Olaya, E. (2010). Estudio descriptivo de los operadores logísticos como componentes estratégicos dentro de la cadena de valor del medicamento en Bogotá. Revista Colombiana de Ciencias Químico Farmacéuticas, 39(2), 168-187. Vallejo, E. \& Camacho, R. (2010). Incidencia de la logística en el marketing. Revista Cientifica Guillermo de Ockham, 8(2), 37-54.

Villarejo Ramos, Á. F. (2002). Modelos multidimensionales para la medición del valor de marca. Investigaciones Europeas en Dirección y Economía de la Empresa, 8(3), 13-44. 